\title{
Seismic Signal Noise Suppression Based on Improved VMD Algorithm
}

\author{
Shen Zijun \\ School of Communication \\ Engineering Chengdu \\ University of Information \\ Technology Sichuan, China
}

\begin{abstract}
Seismic signals contain a variety of attributes, but different attributes are distributed in different frequency bands, so it is necessary to accurately decompose seismic signals into different frequency components, and carry out subsequent denoising processing more effectively. Variational modal decomposition is an effective method for analyzing non-stationary signals. Aiming at the problem of difficult parameter selection of unknown signals, a seismic signal denoising method based on the combination of variational modal decomposition based on quantum particle swarm optimization and Teager energy operator is proposed. The quantum particle swarm optimization algorithm makes the variational modal decomposition algorithm adaptively decompose the signal into eigenmodal functions of different frequency bands, which is convenient for subsequent filtering of signals with different frequencies. In the simulation experiment, the traditional time-frequency analysis method and this method were used to analyze and compare the synthetic signal. According to the comparison results of time-frequency diagram and spectrum diagram, the results show that this method is a more effective seismic signal denoising method..
\end{abstract}

Keywords: variational modal decomposition; Quantum particle swarm optimization; Seismic signal; parameter optimization; Singular spectrum analysis

\section{INTRODUCTION}

Seismic signal is an important source of information for oil and gas exploration. With the reduction of resources, seismic exploration is developing from areas with simple surface conditions to complex mountainous areas, carbonate rock exposure and other complex areas, and the detection target has become complex. Therefore, it is particularly important to eliminate the noise in seismic signal and improve the signalto-noise ratio.

After Huang et al proposed Hilbert Huang transform (HHT) ${ }^{[1]}$ in 1998, this method is widely used. The signal is decomposed into eigenmode components with different frequencies by empirical mode decomposition (EMD), and then Hilbert transform is performed on each eigenmode component to obtain the corresponding time spectrum of the signal. Dragomiretskiy et al. proposed an adaptive and completely non recursive modal variational and signal processing method - variational mode decomposition (VMD) ${ }^{[2]}$ in 2014. By iteratively searching the optimal solution of variational mode, constantly updating each mode function and center frequency, $\mathrm{K}$ eigenmode functions (IMF) with certain bandwidth were obtained, and they were robust to sampling and noise. Wei Liu et al. proposed the use of VMD in seismic signal processing ${ }^{[3]}$, obtained better processing effect compared with EMD and its changes EEMD, CEEMD and ICEEMD, and more effectively highlighted geological characteristics and stratigraphic information in the application of actual data. Liping Zhang et al. ${ }^{[4]}$ proposed that after the seismic data was decomposed into a series of IMF by VMD, the high-frequency IMF was denoised by wavelet transform with soft threshold, and finally the denoised IMF and other IMF were reconstructed to obtain the denoised seismic signal.

However, Hilbert transform has its limitations. When extracting instantaneous frequency and other attributes, it is limited by Bedrosian theorem and Nuttall theorem, and there are some problems such as inaccuracy at both ends. The traditional VMD algorithm needs to subjectively determine the number of decomposed eigenmode components $\mathrm{K}$ and penalty factor alpha, so it is impossible to accurately select appropriate parameters when processing unknown signals. Traditional wavelet denoising depends on the selection of wavelet basis and discrete wavelet transform series. When dealing with unknown signals, it is impossible to accurately select wavelet basis and wavelet transform series through experience, which may lead to the removal of effective components of signals.

To solve these problems, this paper will use the quantum particle swarm optimization algorithm to adaptively optimize the parameters $\mathrm{K}$ and alpha of the variational modal decomposition algorithm, and then use the Teager energy operator to analyze the time-frequency of each processed seismic signal, which effectively increases the stability of the signal time spectrum. Finally, according to the correlation detection principle, the modal components whose correlation is lower than the threshold are denoised by singular spectrum analysis, so as to suppress the random noise of seismic signal.

\section{PRINCIPLE}

\subsection{Quantum particle swarm optimization algorithm}

Particle swarm optimization algorithm was first proposed by Eberhart and Kennedy in $1995^{[5]}$. The algorithm simulated the foraging behavior of bird groups. The solution of each optimization problem was regarded as a bird in the bird group. It was called "particle" in particle swarm optimization algorithm. The flight process of particle was the search process of the individual. Particle had two attributes of position and speed, The speed represented the moving speed, 
the position represented the moving direction, and the particle updated the position and speed in the iterative process to obtain the optimal solution of the optimization problem.

However, in particle swarm optimization algorithm, the particle motion speed is limited, which can not cover the whole solution space, and the convergence speed is slow. From the perspective of quantum mechanics, quantum behavior is applied to particle motion. In quantum mechanics, the motion state of particles with momentum and energy can be expressed by wave function. Therefore, in QPSO model, the wave function is used to represent the motion state of particles. According to Heisenberg uncertainty principle, the position and velocity of particles cannot be accurately measured at the same time. Therefore, QPSO algorithm cancels the moving direction attribute of particles, and the update of particle position has nothing to do with the previous movement of particles, which greatly increases the randomness of particle position, so it can basically cover the whole feasible solution space. The algorithm is usually performed using the following steps:

(1) Initialization: set the maximum number of iterations, the number of objective function arguments and the initial position of particles. Introducing average particle history optimal position: $\frac{1}{M} \sum_{i=1}^{M} P_{\text {best } i}$

Where $\mathrm{M}$ is the population number, $P_{\text {best } i}$ i is the optimal position of the ith particle;

(2) Particle position update: the particle position update formula of quantum particle swarm optimization algorithm can be obtained according to particle swarm optimization algorithm: $P_{i}=\varphi \cdot P_{\text {best_ } i}+(1-\varphi) g_{\text {best }}$;

$x_{i}=P_{i} \pm \alpha\left|M_{\text {best }}-x_{i}\right| \ln \left(\frac{1}{u}\right)$

Where $g_{\text {best }}$ is Optimal position of population, $P_{i}$ updates the position to be used for the ith particle, $\varphi$ Is the wave function of particles, $X_{i}$ is the position of the ith particle.

\subsection{Variational modal decomposition algorithm}

Variational modal decomposition algorithm is a non recursive and adaptive signal decomposition algorithm. The algorithm can determine the number of modal decomposition as needed, and then adaptively match the optimal center frequency and limited bandwidth of each modal component. Therefore, it can effectively separate different frequency components in the signal without modal aliasing. The model of variational modal decomposition is established according to the variational problem with constraints. The variational problem is to ensure that after the original signal is decomposed into $\mathrm{k}$ components, each component has a limited bandwidth of the central frequency, and the sum of the estimated bandwidth of each mode is the smallest. The constraint problem is that the sum of all modal components is equal to the original signal, then the constrained variational problem can be expressed as

$$
\min _{\left\{u_{k}\right\},\left\{\omega_{k}\right\}}\left\{\sum_{k}\left\|\partial_{t}\left[\left(\delta(t)+\frac{j}{\pi t}\right) * u_{k}(t)\right] e^{-j \omega_{k} t}\right\|_{2}^{2}\right\}
$$

$$
\text { s.t. } \sum_{k=1}^{K} u_{k}=f \text {, }
$$

Where $\mathrm{k}$ is the number of modes to be decomposed, $u_{k}$ is the $\mathrm{k}$ modal components by decompose, $\omega_{k}$ Is the center frequency of each mode, $f$ is the seismic signal to be decomposed, $\delta(t)$ is the impact function.

In order to solve the constrained variational problem, a penalty factor is introduced $\alpha$, The Lagrange multiplication operator $\lambda(t)$ can be used to reconstruct the constraint to obtain the augmented function expression:

$L\left(\left\{u_{k}\right\},\left\{\omega_{k}\right\}, \lambda\right)=1 K\left\|\partial_{t}\left[\left(\delta(t)+\frac{j}{\pi t}\right) * u_{k}(t)\right] e^{-j \omega_{k} t}\right\|_{2}^{2}$

$+\left\|f(t)-\sum_{k=1}^{K} u_{k}(t)\right\|_{2}^{2}+\left\langle\lambda(t), f(t)-\sum_{k=1}^{K} u_{k}(t)\right\rangle$

The solution of the augmented Lagrange function is searched by the multiplication operator alternation method (ADMM), and the optimal solution is each eigenmode function in the frequency domain:

$\hat{u}_{k}(\omega)=\frac{\hat{f}(\omega)-\sum_{i \neq k} \hat{u}_{i}(\omega)+(\hat{\lambda}(\omega) / 2)}{1+2 \alpha\left(\omega-\omega_{k}\right)^{2}}$

However, the modal components decomposed by VMD algorithm rely on subjective judgment, and it is difficult to distinguish which component contains more information. Therefore, I propose to use correlation analysis to judge the signal that needs further noise elimination.

\subsection{Singular spectrum analysis}

Singular spectrum analysis is a method for processing nonlinear time series data, which can decompose trend, oscillation component and noise from time series. Its basic idea is to convert the observed time series data into its trajectory matrix:

Where $\mathrm{L}$ is the window length, Calculate $X X^{T}$ and perform singular value decomposition. Let $S=X X^{T}, \lambda_{1}, \lambda_{2}$,

$\ldots, \lambda_{L}$ be the eigenvalue of $\mathrm{S}$, and $\mathrm{u}$ be the standard orthogonal vector of matrix $s$. The singular value decomposition of trajectory matrix $\mathrm{X}$ can be solved as follows:

$X=X_{1}+\cdots+X_{d}, \quad$ Where $X_{i}=\sqrt{\lambda_{i}} U_{i} V_{i}^{T}$

Then, the original time is divided into multiple disjoint groups, and the first $\mathrm{n}$ main components are formed into a new time series according to the demand to realize reconstruction, so as to remove the noise component in the original time series.

\section{APPLICATION}

Firstly, ordinary analog signal was used to verify the algorithm. The analog signal used four time series of different frequencies for superposition, and superimposes $5 \mathrm{~dB}$ noise after synthesis. The analog signal is shown in Figure 1: 


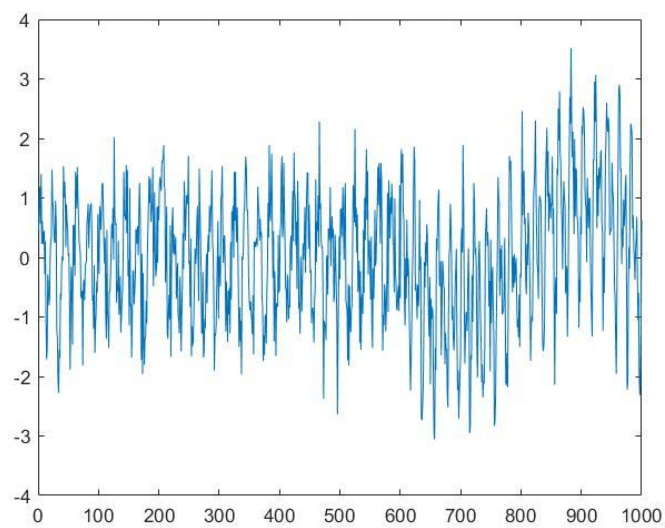

Figure 1.Analog signal

After denoising the signal using the algorithm in this paper, it can be seen from Figure 2 that the processed signal basically restores the frequency components and characteristics without noise.

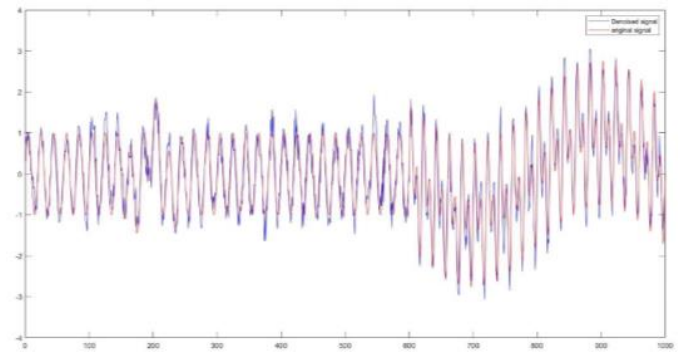

Figure 2.Contrast the denoised signal and the original signal

It can be seen from the time-frequency diagram before and after denoising in figure 3 that basically all high-frequency noise has been successfully filtered, and the original signal has been restored completely.

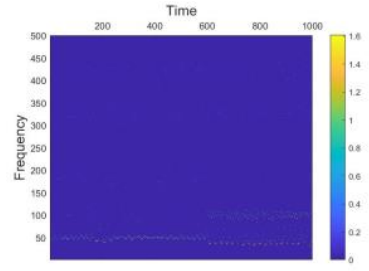

(a)

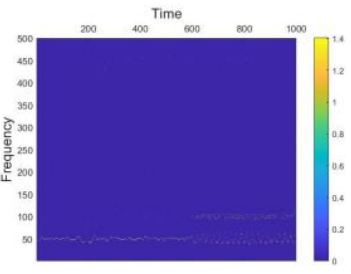

(b)
Figure 3.Example of analog signal

After that, 282 signals was intercepted from the actual seismic data, each signal had 1501 sampling points, and the sampling frequency was $2 \mathrm{~ms}$. We selected one of the signals for analysis. From its time domain image, we could see that the signal burr was significantly reduced after denoising, and then from the frequency comparison diagram, we could see that the high-frequency noise was effectively removed, and the original characteristics of the signal were not greatly affected.

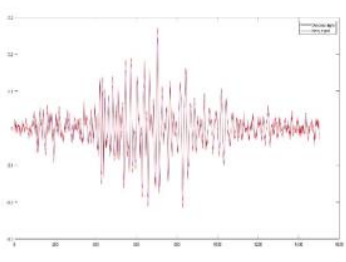

(a)

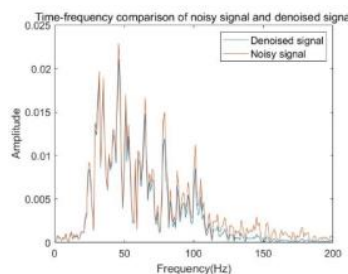

(b)
Figure 4.Example of Actual seismic signal

Next, we denoised the intercepted seismic profile. We could see that the denoised profile could more clearly observe subtle stratigraphic changes in figure 5 , which was helpful for subsequent seismic interpretation such as seismic attribute extraction.

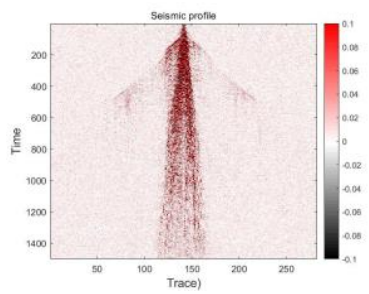

(a)

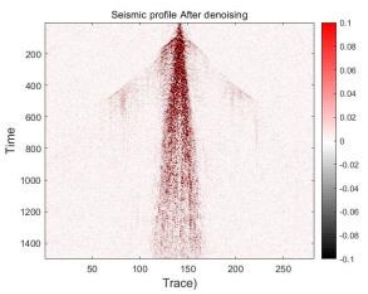

(b)
Figure 5.Example of two dimensional seismic signal

\section{CONCLUSION}

The denoising method based on QPSOVMD-SSA proposed in this paper can effectively remove the high-frequency noise in the seismic signal. Firstly, the seismic signal was processed by QPSOVMD, and then the processed signal was denoised by the improved SSA method, so as to remove the noise in the seismic signal more effectively. This method separated signals of different frequencies, QPSOVMD the loss of lowfrequency signals caused by traditional denoising methods, and the improved VMD algorithm could adaptively obtain the optimal parameters. This is reflected in both analog signals and actual signals, which proves that this method can better denoise seismic signals.

\section{REFERENCES}

[1] Huang N E, Shen Z, Long S R. J. 1998. A new view of nonlinear water waves the Hilbert spectrum. Annual Review of Fluid Mechanics.

[2] Dragomiretskiy,K. J. 2014 Variational Mode Decomposition. TRANSACTIONS ON SIGNAL PROCESSING.

[3] Wei Liu. D. 2016. Research on high resolution timefrequency analysis method and its application in reservoir prediction. China University of Petroleum .

[4] Li-ping Zhang, Ya-juan Xue, Feng Zou, Qiang Chang, De-yong Feng, Jian Zhang. J. 2017. Seismic Signal DeNoising Using Variational Mode Decomposition and Wavelet Transform. International Journal of Petrochemical Science \& Engineering.

[5] Kennedy J,Eberhart R. A. 1995. Particle swarm optimization Proceedings of ICNN'95-International Conference on Neural Networks: IEEE. 